\title{
EFFICACY OF INTRA-MASSETERIC AND SUBMUCOSAL DEXAMETHASONE INJECTION IN SURGICAL EXTRACTION OF IMPACTED LOWER THIRD MOLAR.
}

\footnotetext{
1. BDS, MSc

Senior Registrar Oral Medicine

Sir Syed Dental College, Karachi.

2. BDS, MSc

Lecturer

Department of Oral \& Maxillofacial

Surgery

LUMHS.

3. BDS, FCPS

Assistant Professor Operative

Dentistry

LUMHS

4. BDS, MSc

Lecturer

Department of Oral \& Maxillofacial

Surgery

LUMHS.

Correspondence Address:

Dr. Salman Shams

Lecturer

Department of Oral \& Maxillofacial

Surgery

LUMHS

salman_omfs@hotmail.com

Article received on:

07/07/2018

Accepted for publication:

23/02/2019

Received after proof reading:

25/06/2019
}

\begin{abstract}
Raza Ali', Tahira Shaikh², Mahwish Memon ${ }^{3}$, Salman Shams ${ }^{4}$
ABSTRACT... To determine the efficacy of intra-masseteric and submucosal dexamethasone injection to minimize the postoperative discomfort after the surgical extraction of impacted lower third molar. Study Design: Cross sectional study (Comparative). Setting: Department of Oral \& Maxillofacial Surgery, Institute of Dentistry, Liaquat University of Medical \& Health Sciences Jamshoro / Hyderabad. Period: January 2017 to June 2017. Materials and Methods: All surgical extractions were done under local anaesthesia by giving conventional inferior alveolar nerve block. An envelope mucoperiosteal flap was raised to expose the third molar. Then tooth was extracted. After the removal of tooth any sharp bone was smoothened by bone filer and the socket was washed and sutured. The severity of pain was recorded by using Visual Analogue Scale from 0 (no pain) to 10 (worst pain) described as mild, moderate or severe. Degree of swelling was measured by facial size through Amin and laskin criteria which was measured in millimeters. Mouth opening was measured by interincisal distance through ruler (35-45mm normal value). Results: From sixty four patients it was observed that the minimum age was $28.03+6.12$ years. There were 35 male patients and 29 female patients. When Chi square test was applied, there was a significant association found between Groups (A and B). By using independent sample $t$-test, it was observed that there was a significant association found in both groups concerning facial swelling (Facial size in $\mathrm{mm}$ ) having $\mathrm{p}$-value 0.00 . When the independent t-test was applied on the data, there was a significant association found in Group A and B regarding mouth opening (in $\mathrm{mm}$ ) having p-value 0.001. Conclusions: Dexamethasone has a good efficacy for reducing the postoperative symptoms including severe pain, facial swelling and trismus after the surgical extraction of impacted lower third molar. The better outcomes perceived when it was administered submucosally.
\end{abstract}

Key words: Dexamethasone, Intramasseteric, Lower Third Molar Impaction, Submucosal.

Article Citation: Ali R, Shaikh T, Memon M, Shams S. Efficacy of intra-masseteric and submucosal dexamethasone injection in surgical extraction of impacted lower third molar. Professional Med J 2019; 26(7):000-000.

DOI: 10.29309/TPMJ/2019.26.07.3786

\section{INTRODUCTION}

Impacted tooth is one that fails to erupt into its proper functional occlusion at the age of eruption. ${ }^{1}$ The surgical removal of impacted mandibular third molar is one of the most frequent procedure performed by Oral \& Maxillofacial Surgeon. ${ }^{3}$

The position of an impacted third molar is categorized radiographically using Winter's (Angulation) Classification based on the inclination of the impacted wisdom tooth (3rd molar) to the long axis of the $2^{\text {nd }}$ molar i.e Mesioangular, Distoangular, Vertical or Horizontal impaction. ${ }^{1}$

Indications for mandibular third molar extraction are pain, pericoronitis, periodontal defects, caries, cyst, odontogenic tumor and neurogenic pain. ${ }^{5}$

The surgical removal of impacted $3^{\text {rd }}$ molar can vary in difficulty, degree of trauma to the surgical site and sometimes it require bone removal and soft tissue injury which usually causes significant postoperative pain, swelling and trismus. ${ }^{2,3}$

Many clinical studies are performed to reduce postoperative complications by using corticosteroids, well planned atraumatic surgery, flap design, muscle relaxant and Nonsteroidal anti-inflammatory drugs. $., 5,7,8,9$ 
Masseter is a masticatory muscle which is substantially affected by postoperative edema after impacted mandibular third molar surgery. ${ }^{8}$

Corticosteroids are useful in controlling acute inflammation by interfering with the multiple signaling pathways involved in the inflammatory response. Their primary mechanisms are thought to involve suppression of leukocyte and macrophage accumulation at the site of inflammation, and prevention of prostaglandin formation through the disruption of the arachidonic acid cascade. ${ }^{7,11}$

Dexamethasone, a glucocorticoid has 20-30 times greater potency than natural corticosteroids and long half-life which reduces inflammatory response. ${ }^{6,8}$

Intra-masseteric injection of dexamethasone is easy and tolerable and may be an effective route of administration for reducing postoperative edema, pain and trismus on second postoperative day after lower mandibular third molar surgery its disadvantage is that it is painful, invasive and need expert skill to reach at insertion area. ${ }^{9}$

An alternative to intramasseteric injection is the sub mucosal administration of dexamethasone in buccal vestibule near surgical site, which is simple and easy for both patient and surgeon. 9,10 It is less painful and decreases pain, swelling and trismus which improves patient's quality of life..$^{10,11}$

Submucosal administered dexamethasone is locally administered hence concentrated at surgical site only, whereas intramasseterically administered is spreading systemically. ${ }^{6}$

\section{DATA COLLECTION PROCEDURE}

Patients who fulfilled the inclusion criteria were included in this study. The data was collected from the patients who came to the Out Door Patient department. Informed and written consent was taken from the patient. The impacted tooth was diagnosed by clinical examination and radiograph like OPG and periapical radiographs. like age, gender, preoperative assessment of swelling, pain \& mouth opening were recorded. Patients were divided into two groups Group-A and group-B by Lottery method.

All surgical extractions were done under local anaesthesia by giving conventional inferior alveolar nerve block. An envelope mucoperiosteal flap was raised to expose the third molar. By using straight elevator tooth was lifted up, if tooth was retrieved then procedure was stopped. Otherwise, bone was removed under constant irrigation with sterile $0.9 \%$ normal saline on the occlusal and buccal surface of third molar. Then tooth was extracted.

After the removal of tooth any sharp bone was smoothened by bone filer and the socket was washed with $0.9 \%$ normal saline, then suturing was done with vicryl 3-0 in both groups.

In group A, dexamethasone (inj. Dexamex. Bosch $4 \mathrm{mg}$ ) was given intramasseterically immediately after the flap closure.

In group B, dexamethasone (inj. Dexamex. Bosch $4 \mathrm{mg}$ ) was given submucosally.

Patients was given standard antibiotics for pain relief. Postoperative instructions was given to patient, including soft diet and maintain good oral hygiene by using mouthwash.

The severity of pain was recorded by using Visual Analog Scale from 0 (no pain) to 10 (worst pain) described as mild, moderate or severe. Degree of swelling was measured in millimeters by facial size through Amin and laskin criteria. Mouth opening was measured by interincisal distance through ruler $(35-45 \mathrm{~mm}$ normal value) and all data was recorded on the second and seventh day by the clinician.

\section{RESULTS}

Figure 1 shows Age ranges: the $28.03+6.12$ years was mean age with + standard deviation.

The demographic and clinical parameters 


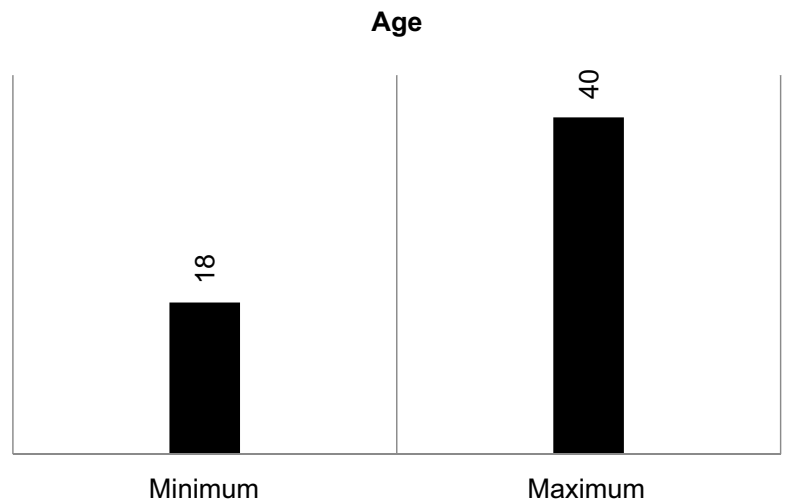

Figure-1. Bar chart representing age ranges

Table-I shows distribution of gender.

\begin{tabular}{|l|c|c|}
\hline & Frequency & Percentage \\
\hline Male & 35 & 54.7 \\
\hline Female & 29 & 45.3 \\
\hline Total & 64 & 100.0 \\
\hline
\end{tabular}

Table-I. Distribution of gender $(n=60)$

Figure-2 shows intensity of pain in group $A$ according to visual analog scale.

Group A (Intramasseteric)

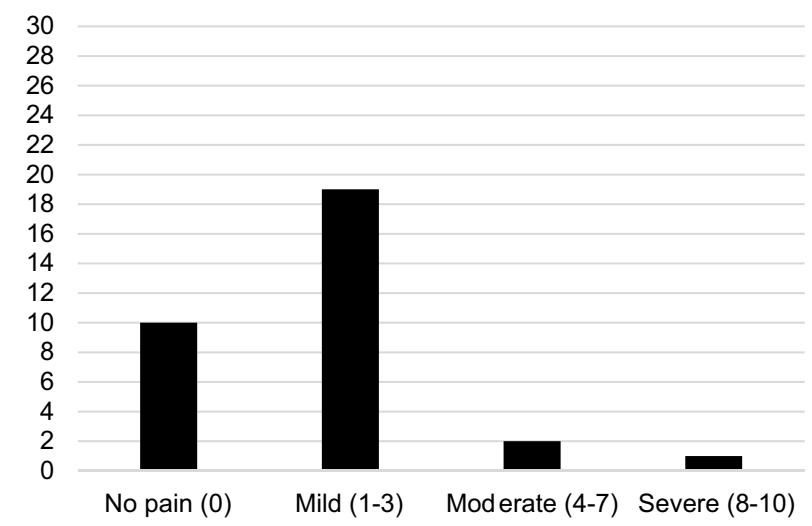

Figure-2. Bar chart representing VAS scale in group A

Figure-3 shows intensity of pain in group B according to visual analog scale.

Table-Il shows comparison of facial swelling.

\begin{tabular}{|l|c|c|c|}
\hline \multicolumn{1}{|c|}{ Group } & n & Mean + SD & P-Value \\
\hline Group A & 32 & $26.70+0.39$ & 0.001 \\
\hline Group B & 32 & $25.60+0.36$ & \\
\hline
\end{tabular}

Table-II. Comparison of swelling (Facial size in $\mathrm{mm}$ ) in both groups $(n=64)$ Independent T-test applied
Group B (Submucosal)

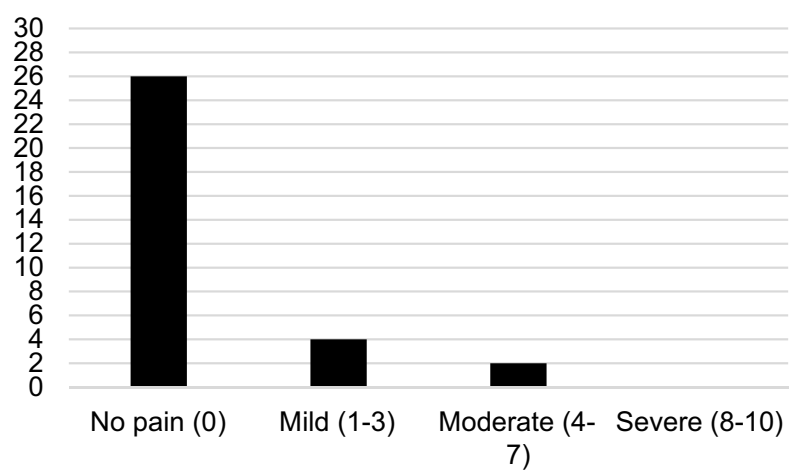

Figure-3. Bar chart representing VAS scale in group B

Table-III shows results about mouth opening in both groups.

\begin{tabular}{|l|c|c|c|}
\hline \multicolumn{1}{|c|}{ Group } & $\mathbf{n}$ & Mean + SD & P-Value \\
\hline Group A & 32 & $38.84+2.38$ & 0.001 \\
\hline Group B & 32 & $41.59+2.87$ & \\
Table-III. Comparison of mouth opening (in $\mathbf{~ m m}$ ) in \\
both groups (n = 64) \\
Independent T-test applied
\end{tabular}

\section{DISCUSSION}

The efficacy of dexamethasone injection at surgery site was depend on the quantity of dose delivering to control the postoperative symptoms, such as facial swelling, severe pain at facial region and limited mouth opening. It has been previously mentioned in many studies that the dexamethasone is act as an inflammatory suppressor and is not only minimize facial swelling, but also reduce pain and trismus after surgical procedure. ${ }^{12}$

A small number of prior studies had objective to investigate the administration of intramasseteric dexamethasone injection to prevent the postoperative complications in lower third molar surgery, however, for third molar surgery in out-patients department, this technique of administrating the steroid injection is frequently used. It has been identified in the intramuscular injection of dexamethasone studies that single dose of dexamethasone could be introduced before or after surgical extraction. This technique could be very effective for better outcomes, however, there is no statistical difference 
between two different quantity of doses, $4 \mathrm{mg}$ or $8 \mathrm{mg} .{ }^{13}$ In another study, it has been specified that the efficacy of dexamethasone could be dependent on the dosage. Moreover, it has been recommended by many surgeons that the 8-12 $\mathrm{mg}$ dexamethasone is injected to achieve the better outcomes. ${ }^{14}$

Several studies have investigated to observe the outcomes of injecting of dexamethasone intramuscular and submucosal with removal wisdom tooth and all these studies have described the varying results ${ }^{15,16[216,217]}$ but one study evaluated that there was no significant difference between submucosal and intramuscular administration.

In another study, it has been observed that the introducing of dexamethasone intramuscularly showed the reduction significantly in postoperative swelling on the first and third days after surgery. It has also been found that pain visual scale analogue scores is significantly decreased too. Additionally, in this study, it has been perceived that the outcomes regarding the postoperative swelling between controls group versus the dexamethasone injection was administered submucosally. In the latter group, significant reduction in swelling has been noticed on the 1 and 3 days after surgery. ${ }^{17}$

While in our present study, when Chi square test was applied to see the effect of intramasseteric and submucosal dexamethasone injections, there was a significant association found between Groups A (intramasseteric) and Group B (submucosal), which indicates that the results of our present study are relevant to international studies.

In few prior study, participants were distributed into three different groups, such as intramuscular, submucosal and control group. In our study, the only two group were suggested to denote precise and definite outcomes between two groups. In our study, the effect of both groups independent sample t-test was applied and it was observed that there was a significant association found in both groups concerning swelling (Facial size in $\mathrm{mm}$ ). Many authors have been used direct measurement method with tape scales and rulers, similar method has been used with this study for the facial swelling because it is simple, reliable and cost-effective method. The postoperative swelling were reduced in both group, but submucosal patients had sufficiently decrease postoperative discomfort and facial swelling than intramasseteric patients. However, it could be difficult to specify that whether the reduction in facial swelling is due to quick systemic dispersion of dexamethasone by intramasseteric or diffusion of dexamethasone in submucosal site at the time of injection.

When the independent sample t-test was applied, there was a significant association found in both Groups concerning mouth opening (in $\mathrm{mm}$ ). There was not much difference between both groups in the respect of mouth opening. As it was considered that the limited mouth opening could be result of facial swelling and surgical trauma. It was clearly reported that there is no significant difference on trismus either administered dexamethasone peri- or post- operatively.

In pervious study, it has been stated that the route of dexamethasone injection submucosally is very effective and easy to delivered technique for minimizing the postoperative discomfort and symptoms including swelling, pain and trismus after the open surgical extraction of impacted lower third molar surgeries. ${ }^{18}$ Dexamethasone is a pharmacological agent, which has a good efficacy to reduce post-surgical third molar removal sequelae, these are commonly as follows: pain, swelling, and trismus. ${ }^{19}$ The introducing of dexamethasone at the third molar surgical region by using submucosal or intra-masseteric techniques showed a greatly decrease in postoperative swelling and pain in the against of control group at all intervals. ${ }^{20}$

\section{CONCLUSION}

It has been ratified on the basis of this research protocols that dexamethasone has a good efficacy for reducing the postoperative symptoms including pain, swelling and mouth opening after the removal of impacted third molar. This study 
also favoured that submucosal administration of dexamethasone is an effective therapeutic technique than intra-masseteric administration technique because submucosal is simple, safe, painless, non-invasive and cost effective method to avoid the moderate to severe postoperative complication of surgical extraction cases of lower third molar.

Copyright@ 23 Feb, 2019.

\section{REFERNCES}

1. Alling CC, Catone GY. Management of impacted teeth. $\mathrm{J}$ oral maxillofac surg, 1993; 51:3-6.

2. Obiechina AE: Update in the technique of third molar surgery. Annals of Ibadan postgraduate medicine 2003; $1: 40-44$.

3. Ferish SE, Bouloux GF. General technique of third molar removal. Oral maxillofac surg clin n Am, 2007; 19:23-43.

4. Karaka I, Simsek S, Uger D. Review of flap design influence on the health of the periodontium after mandibular third molar surgery. Oral surg Oral med Oral pathol Oral radiol, 2007; 104:18-23.

5. Sortino $F$, Cicciù $M$. Strategies used to inhibit postoperative swelling following removal of impacted lower third molar. Dent Res J (Isfahan) 2011; 8:162-71.

6. Laureano Filho JR, Maurette PE, Allais M, Cotinho $M$, Fernandes $C$. Clinical comparative study of the effectiveness of two dosages of dexamethasone to control postoperative swelling, trismus and pain after the surgical extraction of mandibular impacted third molars. Med Oral Patol Oral Cir Bucal 2008; 13:E129-32.

7. Pedersen A. Decadronphosphate in the relief of complaints after third molar surgery. A double-blind, controlled trial with bilateral oral surgery. Int $\mathrm{J}$ Oral Surg 1985; 14:235-40.

8. Neupert EA, Lee JW, Philput CB, Gordon JR. Evaluation of dexamethasone for reduction of postsurgical sequelae of third molar removal. $J$ Oral Maxillofac Surg 1992; 50:1177-82.

9. Alcântara CE, Falci SG, Oliveira-Ferreira F, Santos CR, Pinheiro ML. Pre-emptive effect of dexamethasone and methylprednisolone on pain, swelling, and trismus after third molar surgery: A split-mouth randomized triple-blind clinical trial. Int $\mathrm{J}$ Oral Maxillofac Surg 2014; 43:93-8.
10. Majid OW, Mahmood WK. Effect of submucosal and intramuscular dexamethasone on postoperative sequelae after third molar surgery: Comparative study. Br J Oral Maxillofac Surg 2011; 49:647-52.

11. Majid OW. Submucosal dexamethasone injection improves quality of life measures after third molar surgery: A comparative study. J Oral Maxillofac Surg 2011; 69:2289-97.

12. Dionne RA, Gordon SM, Rowan J, Kent A, Brahim JS. Dexamethasone suppresses peripheral prostanoid levels without analgesia in a clinical model of acute inflammation. Oral Maxillofac Surg 2003; 61:997-1003.

13. Montgomery MT, Hogg JP, Roberts DL, Redding S. The use of glucocorticosteroids to lessen the inflammatory sequelae following third molar surgery. J Oral Maxillofac Surg 1990; 48:179-87.

14. Messer E, Keller J. The use of intraoral dexamethasone after extraction of mandibular third molars. Oral Surg Oral Med Oral Pathol 1975; 40:594-8.

15. Pedersen A. Decadronphosphate in the relief of complaints after third molar surgery. A double-blind, controlled trial with bilateral oral surgery. Int J Oral Surg 1985; 14:235-40.

16. Alexander RE, Throndson RR. A review of perioperative corticosteroid use in dentoalveolar surgery. Oral Surg Oral Med Oral Pathol Oral Radiol Endod 2000; 90:406-15.

17. Majid OW, Mahmood WK. Effect of submucosal and intramuscular dexamethasone on postoperative sequelae after third molar surgery: Comparative study. British J Oral Maxillofac Surg. 2011 31; 49(8):64752.

18. Nair RB, Rahman NM, Ummar M, Hafiz KA, Issac JK, Sameer KM. Effect of submucosal injection of dexamethasone on postoperative discomfort after third molar surgery: A prospective study. J Contemporary Dent Practice. 2013 1; 14(3):401.

19. Vivek GK, Vaibhav N, Shafath A, Imran M. Efficacy of intravenous, intramassetric, and submucosal routes of dexamethasone administration after impacted third molar surgery: A randomized, comparative clinical study. J Adv Clin Res Insights. 2017; 4:3-7.

20. Majid OW. Submucosal dexamethasone injection improves quality of life measures after third molar surgery: A comparative study. J Oral Maxillofaci Surg. 2011 Sep 30; 69(9):2289-97. 


\begin{tabular}{|c|l|l|}
\hline \multicolumn{3}{|c}{ AUTHORSHIP AND CONTRIBUTION DECLARATION } \\
\hline Sr. \# & \multicolumn{1}{|c|}{ Author-s Full Name } & \multicolumn{1}{|c|}{ Contribution to the paper } \\
\hline 1 & Raza Ali & Study concept, Data collection. \\
2 & Tahira Shaikh & Data collection, Literature search. \\
\hline 3 & Mahwish Memon & Data analysis. \\
\hline 4 & Salman Shams & Results, References \\
\hline
\end{tabular}

